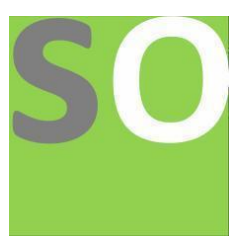

Article title: Multi-receptor mechanism and retinoid signaling disorder in the pathogenesis of COVID-19

Authors: Mahmoud Elkazzaz[1], Tamer Haydara[2], Yousry Esam-Eldin Abo-Amer[3], Israa M Shamkh[4], Mohammed .F. Abo Magd[5], Amr Ahmed[6]

Affiliations: Department of Chemistry and Biochemistry, Faculty of Science, Damietta University, Egypt[1], Faculty of Medicine, Kafrelsheikh University, Egypt[2], Hepatology,Gastroenterology and Infectious Diseases Department, Mahala Hepatology Teaching Hospital, Egypt[3], Chemo and Bioinformatics Lab, Bio Search Research Institution, BSRI. Giza , Egypt, Federal University of Alfenas, Brazil.[4], Zoology and Nematode department, Faculty of Agriculture , Al Azhar University, Cairo, Egypt[5], Director of tuberculosis program Ghubera, public health department ,First health cluster ,Ministry of health , Riyadh, Saudia Arabia.[6]

Orcid ids: 0000-0003-3703-520X[1], 0000-0003-3385-4582[2], 0000-0002-6136-8840[3], 0000-0002-3260-4162[4], 0000-0002-3260-4162[5], 0000-0003-3477-236x[6]

Contact e-mail: mahmoudramadan2051@yahoo.com

License information: This work has been published open access under Creative Commons Attribution License http://creativecommons.org/licenses/by/4.0/, which permits unrestricted use, distribution, and reproduction in any medium, provided the original work is properly cited. Conditions, terms of use and publishing policy can be found at https://www.scienceopen.com/.

Preprint statement: This article is a preprint and has not been peer-reviewed, under consideration and submitted to ScienceOpen Preprints for open peer review.

DOI: 10.14293/S2199-1006.1.SOR-.PPXLYQW.v1

Preprint first posted online: 20 February 2022

Keywords: COVID-19, SARS-CoV-2, Spike protein, STRA6, GPCR, GnRHR, Anosmia, Multi-receptor mechanism, Omicron, C 


\section{Multi-receptor mechanism and retinoid signaling disorder in the pathogenesis of COVID-19}

Mahmud Elkazzaz

1- Department of Chemistry and Biochemistry, Faculty of Science, Damietta University, Egypt

\section{Background}

More than two years have passed since the pandemic and despite all the efforts of researchers, the pathogenesis of COVID-19 has not yet been resolved. Multisystem involvement, neuroendocrine involvement, and pathophysiological changes caused by SARS-CoV-2 in peripheral organs have been shown in many studies. However, the molecular mechanism of these pathophysiological changes caused by COVID-19 has not been elucidated. The frequent mutations of SARS-CoV-2 and the change in the interaction of the virus with host cells have further complicated the pathogenetic mechanism in COVID-19. Unfortunately, the mechanism determined at the beginning of the pandemic and based on the single receptor tropism of ACE2 was insufficient to explain the pathogenesis of COVID-19. It is known that SARS-CoV-2 causes retinoid signaling defects and chemosensory receptor disorders and exerts its pathogenic effects through these mechanisms. Multisystem involvement and different clinical presentations in COVID-19 suggest that virus-host interaction develops through multiple receptors and signaling pathways. The previous mechanism described via ACE2, based on single-receptor tropism, was insufficient to elucidate the pathogenesis of COVID-19 due to the absence of ACE2 receptors in most of the affected organs. In addition, there is no satisfactory explanation for the mechanism by which ACE2free organs are affected in COVID-19. In this regard, we think that ACE2 is not a true binding receptor for the SARS-CoV-2 spike protein. With our recent molecular docking studies, STRA6 and its GPCRs were identified as new binding receptors of the SARS-CoV-2 spike protein. These studies have brought a multi-receptor mechanism to the pathogenesis of COVID-19. The multi-receptor mechanism clearly illuminates the complex pathogenesis of retinoid signaling disorder, systemic organ involvement, neuroendocrine involvement, loss of smell and taste, and many other peripheral symptoms and signs, which are considered an enigma in the pathogenesis of COVID19. Therefore, we suggest retinoid signaling defect as the main pathogenetic disorder in COVID-19 and STRA6 and GPCRs as the main binding receptors of SARS-CoV-2 spike protein. This new mechanism also clarifies the changing symptoms and findings in COVID-19 with each new variant. In addition, these studies have revealed new signaling pathways and drug targets for the treatment and prophylaxis of COVID-19.

KEYWORDS COVID-19, SARS-CoV-2, Spike protein, Omicron, ACE2, STRA6, GPCR, GnRHR, Anosmia, Multireceptor mechanism

\section{Introduction}

Unfortunately, despite the intense efforts of researchers since the beginning of the pandemic, the pathogenesis of COVID-19 has not been clearly resolved. Without solving the mystery here, the chances of developing vaccines and drugs that could be effective against COVID-19 will also be limited. Unfortunately, the pathogenetic mechanism based on cell and tissue tropism provided by ACE2 and TMPRSS2 in COVID-19 cannot clearly meet the systemic organ involvement, neuroendocrine involvement and peripheral organ involvement of COVID-19 (1). It is known that SARS-CoV-2, like many other viruses, enters host cells via receptor-mediated endocytosis and infects host cells in this way. It is also known that SARS-CoV-2 causes chemosensory dysfunction and exerts most of its systemic effects through this mechanism (2). Looking at the big picture, it should be seen that serious systemic effects of SARS-CoV-2 virus and causing damage to many organs are only possible if more than one receptor and signaling pathways organized in different tissues are affected. 
Although ACE2, identified as the major binding receptor in COVID-19, is widely distributed in various human tissues and many of its determinants are well known, organs expressing ACE2 are not equally involved in the pathophysiology of COVID-19 (3). This indicates that other receptors and signaling pathways are involved in the pathogenesis mechanism of virus-host interaction resulting in tissue damage. Although ACE2 expression is frequently seen in the cardiovascular system, lungs, intestines and kidneys, ACE2 expression is absent or very low in the brain, endocrine organs and sensory organs, which are the organs most affected by severe disease $(3,4)$. Moreover, it has been shown that there is no ACE2 expression in other endocrine and peripheral organs, especially in the brain and olfactory neurons, which are most affected by the disease picture in COVID-19 patients $(4,5)$.

Neuroendocrine disorders, hormonal imbalance, olfactory dysfunction, anorexia and retinol deficiency are common symptoms and signs of COVID-19. However, the mechanism of development of these findings cannot be fully explained over ACE2 $(3,5)$. Although there is limited ACE2 expression in some supporting cells in the olfactory epithelium, there is no ACE2 expression in olfactory neurons (5). This shows us that many neuroendocrine disorders, especially the olfactory disorder in COVID-19, develop through some receptors or signaling pathways other than ACE2. The intense expression of STRA6 and GPCR in these organs where there is little or no ACE2 expression suggests that the pathogenetic disorders in COVID-19 develop through these receptors and related signaling pathways. STRA6, GPCRS, and GnRHRs, a type of GPCR receptor, are largely expressed in the brain, endocrine organs, and olfactory neurons $(6,7)$.

Blocking of STRA6 and GPCRs by spike protein with retinoid signaling disorder causes involvement in many other peripheral tissues, especially in neuroendocrine organs $(8,9,10,11)$. Likewise, although the retinoid signaling mechanism and the signal pathways running through STRA6 and its GPCRs seem to be different mechanisms, the retinoid signaling mechanism and the signaling pathways directed by these two receptors are in mutual interaction $(10,11)$. The intense positioning of retinoid signaling $(10,11)$ and GPCRs in the limbic system, which is the center of emotional activity in the brain, and in the thalamus, hypothalamus and pituitary gland, which is the center of neuroendocrine regulation, tells us that the neuroendocrine involvement in COVID-19 shows the retinoid activity disorder in these regions and the spike protein of GPCRs. This suggests that it is due to blocking. SARS-CoV-2 spike protein may cause neuroendocrine, hormonal and metabolic disorders in COVID-19, especially smell and taste defect, by binding to neuroendocrine cells in the brain, olfactory neurons in nasal epithelium and peripheral endocrine tissues via STRA6 and GPCRs $(10,11)$.

Signaling pathways governed by retinoid signaling and STRA6 and its GPCRs are intertwined and interacting mechanisms. Therefore, disruption in the synthesis and functions of STRA6 and GPCRs occurs in the retinoid signaling disorder in COVID-19 $(9,10,11,12)$. The location of STRA6 here is particularly critical, as it facilitates the intracellular transfer of retinol and is at the crossroads of multiple signaling pathways. Likewise, blocking of STRA6 and GPCRs by spike protein may impair retinoid signaling by preventing retinol transfer into the cell $(10,11)$. In COVID-19, the pathogenetic mechanism becomes chaotic due to the fact that many signaling pathways and receptors are affected and their interactions. As a result of these studies, the pathogenetic mechanism in COVID-19 has begun to be clarified with the location of the retinoid signaling defect, STRA6 and GPCRs in the pathogenesis of COVID-19.

ACE2 and multi-receptor mechanism in the pathogenesis of COVID-19 
We think that ACE2 receptors do not have a primary role in the pathogenesis of COVID-19. ACE2 receptors alone are insufficient to explain the pathogenesis of COVID-19. STRA6 and GPCRs play the main role in the pathogenetic disorder caused by virus-host interaction in COVID-19 $(10,11,21)$. Angiotensin converting enzyme 2 or ACE2 for short; It is an enzyme bound to the cell membrane on the outer surface of cells in the heart, lungs, arteries, kidneys and intestines (13). Angiotensin II (AngII), synthesized from Anjitensinogen I as a result of ACE2 activity, causes vasoconstriction by activating Angiotensin receptors (AT1 and AT2), one of the GPCR subtypes $(14,15,16)$. Activated AT2 triggers cellular responses by binding to $G \mathrm{q} / 11$ and $\mathrm{G}$ i/o, respectively from $G$ proteins, activation of phospholipase $C$ and stimulation of protein kinase $C$ via cytosolic $\mathrm{Ca} 2+$. Activated AT receptors also provide inhibition of adenylate cyclase and activation of some tyrosine kinases (17).

The effects of ACE2 are mediated by GPCRs (AT1 and AT2) $(18,19)$. Blocking of ACE2 by the SARS-CoV2 spike protein disrupts G-protein-dependent signaling cascades via AT1 and AT2 receptors, a type of GPCR. The disruption of cellular signaling pathways via AT2 and G proteins by the SARS-CoV-2 bound ACE2 complex still cannot fully explain the severe and systemic effects in COVID-19. Likewise, although AT2 is a G-protein-coupled receptor (20) and displays distinctive motifs and signature residues of G protein-coupled receptors, it fails to display most of the classical features of GPCR signaling $(20,24,25)$.

Another virus-host interaction mechanism operating via ACE2 in COVID-19 is that biological parts of SARS-CoV- 2 can activate AT2 receptors, causing AT2 to bind with ACE2 and disrupt the signaling cascades in this way $(21,26,31)$. There is also an indirect mechanism that operates in this way between SARS-CoV-2, ACE2 and AT2. The third interaction mechanism, which operates through AT2, is the internalization of SARS-CoV- 2 into the cell, which operates through GRK- $\beta$ arrestin. Interestingly, the complex formed by AT2 and GRK- $\beta$ arrest after SARS-CoV-2 binding activates the Gi2/Gi3-proteins, leading to the internalization of the virus-receptor complex into alveolar respiratory epithelial cells (22). As seen here, the internalization of SARS-CoV-2 into the host cell is provided by a mechanism mediated by the AT2 receptor, which is also in the GPCR structure.

Smell and taste disorders in COVID-19 develop with the blocking of STRA6 and GPCRs by the SARS-CoV2 spike protein, as well as systemic organ damage, neuroendocrine disorders, and many peripheral signs and symptoms, as well as retinoid signaling defects $(9,10,11$ th). In addition, ACE2 receptors are very low or absent in the brain, endocrine organs and olfactory neurons, which are the tissues most affected in COVID-19, while their GPCRs have a very intense expression $(23,24,25,29,30)$. In addition, the virus-host interaction that operates via ACE2 in the pathogenesis of COVID-19, the effects of ACE2 are mediated by GPCRs and G proteins. As can be understood from all these, the signaling mechanism operating via ACE2 in virus and host interaction is insufficient to explain the pathogenesis and multisystemic involvement of COVID-19 (23). In this regard, we think that ACE2 is not a true binding receptor for the SARS-CoV-2 spike protein. We propose STRA6 and its GPCRs as major binding receptors of the SARS-CoV-2 spike protein. In addition to ACE2, the identification of GPCR and STRA6 receptors as new binding receptors of spike protein has brought the multi-receptor mechanism to the pathogenesis of COVID-19. Multi-receptor mechanism with retinoid signaling defect, Type I IFN synthesis defect, which is seen as an enigma in the pathogenesis of COVID-19, provides a full explanation for excessive inflammatory cytokine discharge, immune dysregulation, multi-organ involvement, neuroendocrine involvement and peripheral organ involvement.

GPCRs and neuroendocrine disorders in the pathogenesis of COVID-19 
G protein coupled receptors (GPCR) are various membrane receptors and are the largest superreceptor family of genome and signaling proteins (7.25). They also have the nomenclature of "seven transmembrane segments" (7TM) receptors, as they fold through the cell membrane seven times. GPCRs detect extracellular compounds and activate intracellular signal transduction pathways. They do this by binding to $G$ proteins inside the cell (26). Ligands that activate GPCRs include light-sensitive compounds, fragrances, pheromones, hormones, and neurotransmitters. These receptors, which also play a role in many diseases, are the target of approximately $40 \%$ of drugs (27). GPCRs that bind to protein $\mathrm{G}$ i/o have a wide distribution pattern in the human body and are found in almost all organs and tissues $(27,28)$. G i/o-linked GPCRs are especially high in the brain, hematopoietic system, immune system, lung, gastrointestinal system, endocrine organs, and reproductive system, while they are less common in pancreas, fat, and other soft tissues $(7,28)$. It should not be overlooked that the distribution of GPCRs in tissues is parallel to the organ involvement in COVID-19.

More than $90 \%$ of GPCRs are expressed in the brain $(29,30,49)$. They are activated by a wide variety of ligands, including light energy, lipids, sugars, peptides, and proteins $(31,32,33)$ and carry information from the external environment into the cell to mediate relevant functional responses. When ligands bind to GPCRs, they trigger a series of biochemical reactions within the cell by conformational change. These intracellular reactions are involved in sensory functions such as smell, taste, and vision, as well as a wide variety of physiological processes such as secretion, neurotransmission, metabolism, cellular differentiation, inflammation, immune responses, neuronal excitability regulation, metabolism, reproduction, development, hormonal balance, and behavior. controls $(34,35,36)$. G protein-coupled receptors are expressed in many cells in the body. The expression profile shows that GPCRs are expressed in multiple tissues and individual tissues can also express multiple GPCRs $(46,49,50)$. The location of GPCRs as different receptor subunits in many tissues, the presence of different and multiple GPCRs in a single tissue, and their different functions may explain the different clinical signs and symptoms seen in COVID-19 between individuals.

G-protein coupled receptors play a role in many physiological processes. Sight, taste and smell senses, immune system regulation, autonomic nervous system regulation, endocrine and neuroendocrine system regulation, regulation of behavior and mood, perception of cell density, homeostasis, inflammation, tumor growth and metastasis are regulated by the activity of GPCRs $(31,32)$. ,33). G i/olinked GPCRs show intense activity within the nervous system. These receptors participate in complex brain functions such as memory, learning, and cognition by playing a role in neurite outgrowth, neurotransmitter release, and synaptic plasticity (34). We think that many other neurospychiatric symptoms, especially the confused state of consciousness and memory problems, which is called brain fog in COVID19, develop largely due to the blocking of GPCRs in the brain by the spike protein.

To date, about 170 GPCRs have been found to be receptors for intercellular mediators such as hormones and neurotransmitters, and 367 genes have been recognized as GPCR-related genes in the human genome $(37,38)$. Many hormone and neurotransmitter receptors are in the GPCR structure and are named differently in different tissues and cells. Although they interact with different naming and different mediators, all of these receptors act on G proteins. GnRHRs are also a subtype of GPCRs. It has been shown that $\mathrm{G}$-protein coupled receptors are adequately expressed in the hypothalamus, pituitary, limbic system and olfactory region, as well as in the thyroid gland and lung $(37,38)$. GPCRs have also been found to act as thyroxine receptors and are responsible for a variety of biological functions such as appetite regulation, cortisol level, olfaction and taste, as well as intracellular 
transport of retinol $(39,40,41)$. In this regard, we think that in COVID-19, mainly neurological disorders, endocrine disorders such as diabetes, hypothyroidism, adrenal insufficiency, and menstruation and reproductive disorders are caused by the loss of function of these receptors (GPCRs) due to retinoid signaling defect or their blockage by spike protein.

Chemosensory GPCRs (csGPCRs) are essential receptors for sensory signals perceived from outside the body as odor, taste, or pheromone (42). Peptides, lipids, neurotransmitters, and nucleotides are all examples of endogenous signals to which GPCRSs respond (43). The primary function of GPCRs is the conversion of extracellular stimuli into intracellular signals (44). The main feature of GPCRs is that they interact with $\mathrm{G}$ proteins. GPCR receptors affect cellular signaling pathways through G proteins (45). The conformational change that occurs in GPCR with ligand binding leads to the activation of $G$ proteins, allowing the signal to be transferred into the cell. Signaling pathways affected include enzymes such as adenyl cyclase, phospholipase $C$ and D, and ion channels (46). G protein-coupled receptors basically act through two main signaling pathways. These are cyclic adenosine monophosphate (CAMP) and phosphatidylinositol (IP3/DAG) signaling pathways (47). Under normal conditions, inhibitory $\mathrm{G}$ proteins characteristically inhibit adenylate cyclase activity and lower the cAMP concentration (49).

In COVID-19, when the spike protein binds to GPCRs, it creates a conformational change in GPCRs and blocks the binding of GPCRs to inhibitory G proteins. In this way, the signaling pathways that operate through CAMP inhibition and kinase activation may be disrupted. Intracellular cAMP levels may increase with the inhibition of adenyl cyclase inhibition and rapid induction of the CAMP/PKA pathway. Since GPCRs can bind to different isoforms of $\mathrm{G}$ proteins (Gs, Gq/11, Gi, G12/13) in different cells and tissues, spike protein may also affect a number of intracellular alternative signaling cascades (50). Influence of GPCRs on signaling pathways through different isofarms of G proteins in this way may cause different symptoms and findings in COVID-19. This may be the main mechanism underlying the presentation of COVID-19 with different signs and symptoms in a wide range of tissues and organs. The vast clinical presentations and residual diseases in COVID-19 have been the subject of curiosity and research for researchers since the beginning of the pandemic.

\section{STRA6 receptors and retinoid signaling disorder in the pathogenesis of COVID-19}

When STRA6 (Stimulated by retinoic acid 6) was first discovered, it was identified as a cell surface receptor for retinol-binding protein $(51,52)$. However, it was later found to be a transmembrane retinol transporter and a cytokine signal transporter. STRA6 has also been described as the first member of a new protein class called cytokine signal transporter $(51,52)$. A key to the retinoid signaling mechanism and many other cytokine pathways, STRA6 is present at high levels in a variety of tissues, including the choroid plexus, brain microvascular space, facility, spleen, kidney, eye, placenta, and female reproductive system. Surprisingly, however, STRA6 is not found in liver tissue where retinol is primarily stored (51.53). STRA6 mediates cellular uptake of retinol by binding with the retinol-coupled RBP molecule to trigger the release and internalization of retinol, in addition to cholesterol uptake into human cells (53). It has been shown that both retinol and cholesterol decrease in COVID-19 infection $(54,55,56,57)$. 
STRA6 is at the center of a complex signaling network and plays a key role $(58,59)$. After the retinolRBP complex binds to STRA6, it activates the JAK/STAT pathway, enabling the activation of the STAT5 transcription factor. The retinol transporter function of STRA6 and its cytokine signaling receptor function are interdependent processes and interact with each other (59). While activation of STRA6 with JAK2 is required for retinol uptake, STRA6 signal can inhibit JAK2 signal through SOCS3 $(60,61)$. While this interaction regulates the signaling pathways, it also manages the controlled uptake of retinol into the cell. SOCS3, the first protein classified as a cytokine signal suppressor, also played a role in this regulation. SOCS3 inhibits JAK2 activity by binding to JAK2 kinase $(61,62)$. In this respect, STRA6 is also associated with some other cytokine signal suppressors, especially SOCS3. Considering all this, it is likely that STRA6 functions as a vitamin A receptor, transmembrane retinol transporter, and cytokine signal transporter as well as a cytokine signal suppressor. Blocking of STRA6 by spike protein in COVID19 may disrupt the cytokine signal suppression function of STRA6 and SOCS3, leading to a signaling disorder that may lead to disruption of Type I interferon synthesis in the pathogenesis of COVID-19 (62).

STRA6 determines cell fate by integrating retinoid signaling with numerous signaling pathways such as p53, MAPK/Erk, PI3K/Akt, mTOR, Jak/STAT, Ca-Calmodulin, Wnt/catenin $(9,10,65)$. It should not be overlooked that most of these pathways are related to transcription mediators such as STATs and NFkB, which are essential regulatory mediators for vital functions such as inflammatory cytokine release, apoptosis, cell cycle and cell survival. It has been found that excessive activity of the STAT pathway in COVID-19 is associated with severe clinical pictures (63). STRA6 expression and activity depend on retinoid activity (64). Likewise, adequate and balanced activity of STRA6 is required for normal retinoid activity. STRA6 activity and retinoid signaling activity interact with each other (64).

Retinoid signaling disorder is at the center of the pathogenesis of COVID-19 and causes severe systemic effects in COVID-19 (9). Excessive use of retinol in the synthesis of Type I interferon and suppression of inflammation in COVID-19 causes retinol depletion and retinoid signaling defect (9). In addition, the blocking of the retinol receptor and the STRA6 receptor, which is the transmembrane transporter of retinol, by the spike protein also disrupts the intracellular retinol transfer, leading to a decrease in endogenous retinoic acid levels and retinoid signaling disorder $(9,19)$. As a result, disruption in many signaling pathways and immune system response in COVID-19, Type I interferon synthesis defect and an increase in inflammatory cytokine synthesis and release via the UPS/NFkB system occur $(8,9,10)$.

Disruption of many cellular signaling pathways operating through STRA6 causes dramatic clinical manifestations in COVID-19. Activation of the MAPK/ERK2, AKT, C-MYC or PI3K/AKT pathway via RARa leads to significant changes in cellular differentiation, proliferation and tumorigenesis $(9,10,67)$. Signaling pathways working depending on RARA activity are highly active signaling cascades in many cells, especially in the nervous system and immune system (68). RAR $\alpha$, a subtype of RARs, is localized in membrane lipid rafts, such as STRA6, and similarly activates cytoplasmic kinases after binding to the RA ligand, leading to the activation of various intracellular signaling pathways, including PI3K/Akt (68). Signaling pathways such as MAPK/Erk, Jak/STAT, RIG-I, mTOR, GluR1 and Ca-Calmodulin, which are dependent on STRA6 and RARA activity, are highly active signaling cascades in many cells, especially in the nervous system and immune system $(69,70)$. . Under normal conditions, GPCR signal inhibits mTORC1 through PKA phosphorylation $(66,69)$. Blocking this signaling mechanism by spike protein in COVID-19 will impair mTOR inhibition via PKA, and cell growth, metabolism and autophagy governed by $\mathrm{mTORC} 1$ will also be impaired (71). 
In COVID-19, genomic and nongenomic effects controlled by retinoic acids are particularly impaired due to retinoid signaling disorder (68). Activation of functional proteins and enzymes through protein kinase activity is provided by retinoid signaling $(69,70)$. With the disruption of the retinoid signaling mechanism, the synthesis and activation of functional proteins and enzymes are disrupted and cellular functions are also impaired $(69,70)$. If there is enough RA in the cell, kinase activity, protein phosphorylation and associated signaling pathways continue to operate $(9,66)$. In COVID-19, kinase activity and protein phosphorylation are disrupted due to decreased endogenous RA levels and retinoid signaling disorder. When the kinase activity provided by retinoid activity is impaired, Type I IFN synthesis, which occurs through the phosphorylation of IFRs, is particularly impaired $(9,10,54)$.

Type I interferon synthesis occurs through a mechanism in which the genomic and nongenomic activities of RAs are carried out together $(9,54)$. While the phosphorylation of IRFs is provided by the nongenomic activity of RAs, the activation of genes responsible for Type I interferon synthesis via transcription factors is provided by the genomic activity of RAs $(8,9)$. Another mechanism by which Type I interferon synthesis is disrupted in COVID-19 is that the viral proteins of SARS-CoV-2, NSP5, ORF6 and ORF8, block the displacement of STATs or inhibit the phosphorylation of mediator molecules, thereby inhibiting Type I IFN synthesis and JAK-STAT. occurs by disrupting the signaling (72). An imbalance in JAK-STAT signaling has been found to be associated with COVID-19 severity (73). Type I IFN strengthens cellular and humoral immune responses, working like a conductor in regulating the immune system in host defense. Type I IFN synthesis defect in COVID-19 is one of the most important pathophysiological disorders that aggravate the clinical picture together with inflammatory cytokine discharge and has been shown in many studies $(8,9,84)$.

It was found that cAMP-dependent protein kinase (PKA) activity was rapidly up-regulated in NB4 cells treated with the retinol derivative ATRA. Phosphorylation of RARa by PKA via ATRA increases transactivation of RARa $(67,68)$. Decreased RA levels impair kinase activity and phosphorylation of RARA by PKA $(67,68,69)$. GluR1 is a presynaptic receptor and provides the transmission of the sense of smell via glutamate. GluR1 synthesis occurs with the nongenomic effect of RAs and RARa activity in the cytoplasmic compartment (68). Decreased endogenous RA levels cause disruption of RARa phosphorylation, disruption of GluR1 synthesis, and loss of sense of smell in COVID-19 (8,9). In addition to the decrease in the synthesis and activity of GluR1 and GPCRs in COVID-19, the blocking of GPCRs in olfactory neurons by the spike protein is also responsible for olfactory loss (11).

There is also a strong correlation between retinoid signaling and GPCRs. Like STRA6 receptors, GPCRs are synthesized depending on retinoic acids (74). Therefore, the retinoid signaling defect causes the synthesis defect of STRA6 and GPCRs as well as the disruption of signaling pathways controlled by STRA6 and GPCRs. With the disruption of signaling pathways related to STRA6 and GPCRs, serious disruptions occur in the cellular cycle, neuroendocrine system, apoptosis mechanism, immune system regulation and suppression of inflammation (75). In COVID-19, retinoid signaling defect specifically impairs neuronal conduction and neuronal plasticity $(9,75)$. The different location of GPCRs in the brain, endocrine organs and peripheral tissues and their ability to bind to different $\mathrm{G}$ protein isoforms may cause organ-specific symptoms and signs of COVID-19 (76). Since the blockade of the STRA6 pathway in COVID-19 causes retinoid signaling defect, we think that disruption of this pathway may be responsible for severe systemic findings in COVID-19, and that the blocking of GPCRs may be responsible for the emergence of some neuroendocrine disorders and organ-specific signs and symptoms. 


\section{Intracellular internalization of SARS-CoV-2 and GPCRs in COVID-19}

Although GPCRs are important receptors of signal-transmitting lipid mediators, they also played an important role in the internalization of SARS-CoV-2 into the cell via the adenosine-CFTR system (22). Viruses effectively use intracellular signaling pathways for replication and life cycle. Like most viruses, SARS-CoV-2 is taken up by endocytosis via membrane surface receptors to enter host cells (23). It is known that SARS-CoV-2 carries an outer lipid membrane and adheres to host cells with a lipoprotein spike protein embedded in this membrane. However, even if SARS-CoV-2 binds to the ACE2 receptor, it can enter host cells by indirect mechanisms including GPCRs $(22,77)$.

Under physiological conditions, the adenosine-CFTR regulation system is critical for mucosal airway surface protection and regulation of the alveolar surface layer (78). However, in COVID-19, CFTR is also involved in the uptake of SARS-CoV-2 into the host cell together with GPCRs $(22,77,78)$. It is also known that the ACE2-AT2 (GPCR) complex formed by the binding of SARS-CoV-2s to ACE2 is taken into respiratory alveolar epithelial cells by catalyzing the activation of Gi2/Gi3-proteins over GRK- $\beta$ arrestin $(22,79)$. Based on current findings, SARS-CoV-2 uses three distinct pathways via GPCR to enter host cells. The first of these is the internalization of the spike protein into the host cell via AC2 via AT2, and the other two pathways are provided by the mechanisms provided through the Adenosine-CFTR system and the AT2-GRK $\beta$ arrestin system.

Although ACE2 appears to be the primary receptor for the entry of SARS-CoV-2 into the cell, it is clearly seen that the mechanism operates through the AT2 receptors in the GPCR structure. Recognition of intracellular viruses by the host immune system is provided by retinoic acid-inducible gene-I (RIG-I), which is the most important cytosolic viral RNA recognizer that initiates the congenital immune system response against cytosolic RNA viruses (80). Interestingly, RIG-I was initially identified as an orphan Gprotein coupled receptor induced by retinoic acid and whose ligand is unknown, but later it was determined that viral triphosphate-RNA ends were ligands of RIG-I (81). It is clear that the activity of RIG-I, which is synthesized due to retinoic acid activity, will also decrease due to retinol depletion and retinoid signaling defect. Therefore, the retinoid signaling defect in COVID-19 also renders the RIG I pathway, which is one of the important pathways responsible for type I interferon synthesis, nonfunctional $(82,83)$.

\section{Omicron variant and multi-receptor mechanism}

Each new mutation in SARS-CoV-2 causes conformational changes in the spike protein and changes the binding state of the SARS-CoV-2 spike protein to ACE2, STRA6 and GPCR receptors. The conformational change in the spike protein also causes a change in the receptor binding site of the spike protein, resulting in a change in the affinity of the spike protein to ACE2, STRA6 and GPCRs, and binding to these receptors with different strengths or impaired binding to these receptors. Since this affinity and binding change between the spike protein and the host cells also affects the cellular signaling pathways managed by the receptors, it causes a change in clinical signs and symptoms with each new variant in patients.

Omicron, the last variant of SARS-CoV-2 and now the dominant strain on the world, was found to bind to ACE2 receptors twice as strongly compared to previous variants (85). This increase in binding strength caused the virus to spread more quickly and spread the epidemic again. Despite this, severe clinical pictures have started to be seen less frequently with the Omicron variant $(84,86)$. This situation 
also shows that ACE2 does not play a leading role in the pathogenesis of COVID-19 and the aggravation of the clinical picture. With the new mutation, the increase in the binding power of the spike protein to ACE2 and the intense expression of ACE2 in the upper respiratory tract mucosa were effective in the increased colonization of Omicron in the respiratory tract and increased transmission $(84,87)$.

\section{Conclusion}

The complex pathogenetic mechanism, multisystemic involvement, and different clinical presentations in COVID-19 make us think that the SARS-CoV-2 spike protein interacts with other receptors besides ACE2 in COVID-19. The pathophysiological changes caused by SARS-CoV-2 in the central nervous system and peripheral organs have been shown in many studies. However, the molecular mechanism of these pathophysiological disorders has not been elucidated. The frequent mutations of SARS-CoV-2 and the change in the interaction of spike protein with host cells have complicated the pathogenesis of COVID-19. The pathogenetic view, which was determined at the beginning of the pandemic and based on the single receptor mechanism of ACE2, was unfortunately insufficient to explain the pathogenetic mechanism underlying the severe and systemic involvement of COVID-19.

Previous studies have shown that retinol decreases in COVID-19 in correlation with the clinical picture and is depleted in severe patients. Retinoid signaling disorder that develops with retinol depletion in COVID-19 causes dysregulation in the immune system. The main cause of Type I interferon synthesis defect and excessive inflammatory cytokine discharge in COVID-19 is the dysregulation of the immune system caused by retinoid signaling disorder. This finding also clearly meets the biphasic immunopathogenesis in COVID-19, on which the researchers agree (88). Blocking of the STRA6 receptor by the spike protein disrupts the intracellular transfer of retinol, resulting in decreased endogenous retinoic acid levels and retinoid signaling defect. The blockade of the STRA6 receptor, which is at a junction point in the cellular signaling mechanism, also disrupts important signaling pathways such as janus kinase, mTOR, PI3K/akt, Raf/Mek/Erk, ca-calmodulin and STAT, in addition to the retinoid signal. It is clear that severe systemic effects in COVID-19 occur with the blocking of the STRA6 receptor by the spike protein and the disruption of the signaling pathways provided by STRA6.

Retinol depletion and retinoid signaling defect caused by spike protein blockade of the STRA6 receptor appears to be responsible for the severe and systemic manifestations in COVID-19. In addition, we think that with the blocking of GPCRs by the SARS-CoV-2 spike protein, symptoms and findings mostly occur in the neuroendocrine regulation center and peripheral endocrine organs. Blocking of GPCRs by spike protein can explain the wide spectrum of diseases in COVID-19 because GPCRs are located in many tissues, affect many neurotransmitters and signaling pathways, and act on different G-protein isotypes.

In conclusion, although ACE2 has been identified as the main binding receptor of the SARS-CoV-2 spike protein for host cells, it is understood that ACE2 does not have a primary role in the pathogenesis of COVID-19 and aggravation of the clinical picture. In the pathogenesis of COVID-19, SARS-CoV-2 disrupts cellular signaling pathways by blocking STRA6 and GPCRs of spike protein and invades host cells. Potentially, acute, chronic neurological, endocrine and metabolic signs and symptoms of SARS-CoV-2 occur with the disruption of cellular signaling pathways working due to STRA6 and GPCRs. In COVID19 , devastating systemic involvement and neuroendocrine disorders, olfactory dysfunction, loss of appetite, retinol deficiency, retinoid signaling defect and other metabolic disorders develop through STRA6 and GPCRs. Therefore, we think that the blocking of STRA6 and GPCRs by spike protein plays an 
important role in the pathogenesis of COVID-19 and can be used as potential therapeutic targets for the treatment and prophylaxis of COVID-19.

\section{Conflicts of interest}

The authors have noconflicts of interest to declare that are relevant to the content of this article.

\section{Funding}

None

\section{References}

1. Soni S, Jiang Y, Tesfaigzi Y, Hornick JL, Çataltepe S. Comparative analysis of ACE2 protein expression in rodent, non-human primate, and human respiratory tract at baseline and after injury: A conundrum for COVID-19 pathogenesis. PLoS One. 2021 Feb 24;16(2):e0247510.

2. Zeng M, Wang DY, Mullol J, Liu Z. Chemosensory Dysfunction in Patients with COVID-19: What Do We Learn from the Global Outbreak? Curr Allergy Asthma Rep. 2021;21(2):6. Published 2021 Feb 3.

3. Hamming I, Timens W, Bulthuis ML, Lely AT, Navis G, van Goor H. Tissue distribution of ACE2 protein, the functional receptor for SARS coronavirus. A first step in understanding SARS pathogenesis. J Pathol. 2004 Jun;203(2):631-7.

4. Bourgonje AR, Abdulle AE, Timens W, Hillebrands JL, Navis GJ, Gordijn SJ, Bolling MC, Dijkstra G, Voors AA, Osterhaus AD, van der Voort PH, Mulder DJ, van Goor H. Angiotensin-converting enzyme 2 (ACE2), SARS-CoV-2 and the pathophysiology of coronavirus disease 2019 (COVID-19). J Pathol. 2020 Jul;251(3):228-248.

5. Brann DH, Tsukahara T, Weinreb C, Lipovsek M, Van den Berge K, Gong B, Chance R, Macaulay IC, Chou HJ, Fletcher RB, Das D, Street K, de Bezieux HR, Choi YG, Risso D, Dudoit S, Purdom E, Mill J, Hachem RA, Matsunami H, Logan DW, Goldstein BJ, Grubb MS, Ngai J, Datta SR. Non-neuronal expression of SARS-CoV-2 entry genes in the olfactory system suggests mechanisms underlying COVID19-associated anosmia. Sci Adv. 2020 Jul 31;6(31):eabc5801.

6. Billington CK, Penn RB. Signaling and regulation of G protein-coupled receptors in airway smooth muscle. Respir Res. 2003;4(1):2.

7. Schoneberg, T., Schulz, A., Biebermann, H., Hermsdorf, T., Rompler, H., and Sangkuhl, K. (2004). Mutant G-protein-coupled receptors as a cause of human diseases. Pharmacol. Ther. 104 (3), 173-206.

8. Sarohan A.R. COVID-19: endogenous retinoic acid theory and retinoic acid depletion syndrome. Med. Hypotheses, 144 (2020), Article 110250

9. Sarohan AR, Kızıl M, İnkaya AÇ, Mahmud S, Akram M, Cen O. A novel hypothesis for COVID-19 pathogenesis: Retinol depletion and retinoid signaling disorder. Cell Signal. 2021 Nov;87:110121.

10. Elkazzaz MR, Haydara T, Abo-Amer YE, et al. Discovery of the Retinol STRA6 Receptor as a Novel Binding Receptor for SARS-CoV-2 in COVID-19: In Silico Research. Research Square; 2021. 
11. Mahmoud Ramadan Elkazzaz, Amr Ahmed, Ghareeb Alshuwaier, Israa M Shamkh, Yousry EsamEldin Abo-Amer, Tamer Haydara. In silico discovery of GPCRSs and GnRHRs as Novel SARS-CoV-2 binding receptors, the Scientic Breakthrough that could explain the observed High cortisol, Appetite loss, Ansomnia and Hypogonadism, as well as Hypothyroidism, Retinol deciency and menstrual disturbances among SARS-COV-2 patients. https://www.researchsquare.com/article/rs-1155356/v3. Posted Date: December 27th, 2021. DOI: https://doi.org/10.21203/rs.3.rs-1155356/v3

12. Singh Y, Gupta G, Satija S, Pabreja K, Chellappan DK, Dua K. COVID-19 transmission through host cell directed network of GPCR. Drug Dev Res. 2020;81(6):647-649. doi:10.1002/ddr.21674

13. Gene: ACE2, angiotensin I converting enzyme 2. National Center for Biotechnology Information (NCBI).U.S. National Library of Medicine. 28 February 2020. Archived from the original on 24 December 2014. Accessed on April 15, 2020.

14. Deshpande DA, Wang WC, Mcllmoyle EL, Robinett KS, Schillinger RM, An SS, Sham JS, Liggett SB. Bitter taste receptors on airway smooth muscle bronchodilate by localized calcium signaling and reverse obstruction. Nat Med. 2010 Nov;16(11):1299-304.

15. Donoghue M, Hsieh F, Baronas E, Godbout K, Gosselin M, Stagliano N, Donovan M, Woolf B, Robison $\mathrm{K}$, Jeyaseelan R, Breitbart RE, Acton S. A novel angiotensin-converting enzyme-related carboxypeptidase (ACE2) converts angiotensin I to angiotensin 1-9. Circ Res. 2000 Sep 1;87(5):E1-9.

16. Keidar S, Kaplan M, Gamliel-Lazarovich A. ACE2 of the heart: From angiotensin I to angiotensin (17). Cardiovasc Res. 2007 Feb 1;73(3):463-9.

17. Wang W, McKinnie SM, Farhan M, Paul M, McDonald T, McLean B, Llorens-Cortes C, Hazra S, Murray AG, Vederas JC, Oudit GY. Angiotensin-Converting Enzyme 2 Metabolizes and Partially Inactivates Pyr-Apelin-13 and Apelin-17: Physiological Effects in the Cardiovascular System. Hypertension. 2016 Aug;68(2):365-77.

18. Higuchi S, Ohtsu H, Suzuki H, Shirai H, Frank GD, Eguchi S (April 2007). "Angiotensin II signal transduction through the AT1 receptor: novel insights into mechanisms and pathophysiology". Clinical Science. 112 (8): 417-28.

19. Porrello ER, Delbridge LM, Thomas WG. The angiotensin II type 2 (AT2) receptor: an enigmatic seven transmembrane receptor. Front Biosci (Landmark Ed). 2009 Jan 1;14:958-72.

20. Clayton D, Hanchapola I, Thomas WG, Widdop RE, Smith AI, Perlmutter P, Aguilar MI. Structural determinants for binding to angiotensin converting enzyme 2 (ACE2) and angiotensin receptors 1 and 2. Front Pharmacol. 2015 Jan 30;6:5.

21. Yogendra Singh,Gaurav Gupta,Saurabh Satija,Kavita Pabreja,Dinesh Kumar Chellappan,Kamal Dua. COVID-19 transmission through host cell directed network of GPCR. Drug Development Research 23 April 2020.

22. Manglik, A., Wingler, LM, Rockman, HA \& Lefkowitz, RJ beta-Arrestin-Biased Angiotensin II Receptor Agonists for COVID-19. Circulation 142, 318-320 (2020). 
23. Hopkins C, Lechien JR, Saussez S. More that ACE2? NRP1 may play a central role in the underlying pathophysiological mechanism of olfactory dysfunction in COVID-19 and its association with enhanced survival. Med Hypotheses. 2021 Jan;146:110406

24. Francois Alhenc-Gelas, Tilman B. Drueke. Blockade of SARS-CoV-2 infection by recombinant soluble ACE2. NEPHROLOGY DIGEST COVID-19| VOLUME 97, ISSUE 6, P1091-1093, JUNE 01, 2020. Published: April 14, 2020.

25. Penn RB, Benovic JL. Regulation of heterotrimeric $G$ protein signaling in airway smooth muscle. Proc Am Thorac Soc. 2008 Jan 1;5(1):47-57.

26. Trzaskowski B, Latek D, Yuan S, Ghoshdastider U, Debinski A, Filipek S. Action of molecular switches in GPCRs--theoretical and experimental studies. Curr Med Chem. 2012;19(8):1090-1109.

27. Filmore D (2004). It's a GPCR world. Modern Drug Discovery. American Chemical Society. 2004 (November): 24-28.

28. Overington JP, Al-Lazikani B, Hopkins AL, December 2006. How many drug targets are there? Nature Reviews. Drug Discovery. 5 (12). ss. 993-6.

29. Doggrell SA. Bitter taste receptors as a target for bronchodilation. Expert Opin Ther Targets. 2011 Jul;15(7):899-902. doi: 10.1517/14728222.2011.580279. Epub 2011 Apr 27. PMID: 21521131.

30. Vassilatis, Demetrios $\mathrm{K}$ et al. The $\mathrm{G}$ protein-coupled receptor repertoires of human and Mouse. Proceedings of the National Academy of Sciences of the United States of America vol. 100,8 (2003): 4903-8.

31. Pera T, Hegde A, Deshpande DA, Morgan SJ, Tiegs BC, Theriot BS, Choi YH, Walker JK, Penn RB. Specificity of arrestin subtypes in regulating airway smooth muscle $G$ protein-coupled receptor signaling and function. FASEB J. 2015 Oct;29(10):4227-35.

32. Thompson MD, Percy ME, McIntyre Burnham W, Cole DE. G protein-coupled receptors disrupted in human genetic disease. Methods Mol Biol. 2008;448:109-37.

33. Lundstrom, K. (2009). An overview on GPCRSs and drug discovery: structure-based drug design and structural biology on GPCRSs. Methods Mol. Biol. 552, 51-66.

34. Lagerström, M. C., and Schiöth, H. B. (2008). Structural diversity of G protein-coupled receptors and significance for drug discovery. Nat. Rev. Drug Discov. 7 (4), 339-357.

35. Rosenbaum, D. M., Rasmussen, S. G. F., and Kobilka, B. K. (2009). The structure and function of Gprotein-coupled receptors. Nature 459 (7245), 356-363.

36. Venkatakrishnan, A. J., Deupi, X., Lebon, G., Tate, C. G., Schertler, G. F., and Babu, M. M. (2013). Molecular signatures of G-protein-coupled receptors. Nature 494, 185.

37. Ahmad, R., Wojciech, S., and Jockers, R. (2015). Hunting for the function of orphan GPCRSs - beyond the search for the endogenous ligand. Br. J. Pharmacol. 172 (13), 3212-3228. 
38. Gloriam DE, Fredriksson R, Schiöth HB. The G protein-coupled receptor subset of the rat genome. BMC Genomics. 2007 Sep 25;8:338.

39. D.S. Im. Orphan G protein-coupled receptors and beyond. Jpn. J. Pharmacol., 90 (2002), pp. 101106

40. Buck L, Axel R. A novel multigene family may encode odorant receptors: a molecular basis for odor 54- recognition. Cell. 1991 Apr 5; 65(1):175-87.

41. Firestein S. The good taste of genomics. Nature. 2000 Apr 6;404(6778):552-3.

42. Howard AD, McAllister G, Feighner SD, Liu Q, Nargund RP, Van der Ploeg LH, Patchett AA. Review Orphan G-protein-coupled receptors and natural ligand discovery. Trends Pharmacol Sci. 2001 Mar; 22(3):132-40.

43. Lee DK, George SR, Evans JF, Lynch KR, O'Dowd BF. Review Orphan G protein-coupled receptors in the CNS. Curr Opin Pharmacol. 2001 Feb; 1(1):31-9.

44. Kroeze WK, Sheffler DJ, Roth BL. G-protein-coupled receptors at a glance. J Cell Sci. 2003 Dec 15;116(Pt 24):4867-9.

45. Lundstrom K. The future of $\mathrm{G}$ protein-coupled receptors as targets in drug discovery. IDrugs. 2005 Nov;8(11):909-13.

46. Thompson MD, Cole DE, Jose PA. Pharmacogenomics of $G$ protein-coupled receptor signaling: insights from health and disease. Methods Mol Biol. 2008;448:77-107.

47. Gilman AG (1987). G proteins: transducers of receptor-generated signals. Annual Review of Biochemistry. 56 (1). ss. 615-49.

48. Huber T, Menon S, Sakmar TP. Structural basis for ligand binding and specificity in adrenergic receptors: implications for GPCR-targeted drug discovery. Biochemistry. 2008 Oct 21;47(42):11013-23.

49. de Oliveira PG, Ramos MLS, Amaro AJ, Dias RA, Vieira SI. Gi/o-Protein Coupled Receptors in the Aging Brain. Front Aging Neurosci. 2019 Apr 24;11:89.

50. Zhou Q, Yang D, Wu M, et al. Common activation mechanism of class A GPCRs. Elife. 2019;8:e50279. Published 2019 Dec 19.

51. Blaner WS (March 2007). "STRA6, a cell-surface receptor for retinol-binding protein: the plot thickens". Cell Metabolism. 5 (3): 164-6.

52. Ruiz A, Mark M, Jacobs H, Klopfenstein M, Hu J, Lloyd M, Habib S, Tosha C, Radu RA, Ghyselinck NB, Nusinowitz S, Bok D (May 2012). "Retinoid content, visual responses, and ocular morphology are compromised in the retinas of mice lacking the retinol-binding protein receptor, STRA6". Investigative Ophthalmology \& Visual Science. 53 (6): 3027-39.

53. Kawaguchi R, Yu J, Honda J, Hu J, Whitelegge J, Ping P, Wiita P, Bok D, Sun H (February 2007). "A membrane receptor for retinol binding protein mediates cellular uptake of vitamin A". Science. 315 (5813): 820-5. 
54. Aziz Rodan Sarohan, Hakan Akelma, Eşref Araç, Özgür Aslan "Retinol Depletion in Severe COVID19 "medRxiv 2021.01.30.21250844; doi: https://doi.org/10.1101/2021.01.30.21250844.

55. Tepasse, P.-R.; Vollenberg, R.; Fobker, M.; Kabar, I.; Schmidt, H.; Meier, J.A.; Nowacki, T.; HüsingKabar, A. Vitamin A Plasma Levels in COVID-19 Patients: A Prospective Multicenter Study and Hypothesis. Nutrients 2021, 13, 2173. https://doi.org/10.3390/nu13072173

56. Bilge M, Kibar Akıllı I. Low-Density Lipoprotein Cholesterol Levels and Disease Severity in COVID-19 Pneumonia. Med J Bakirkoy 2021;17:274-279

57. Zhao M, Luo Z, He H, et al. Decreased Low-Density Lipoprotein Cholesterol Level Indicates Poor Prognosis of Severe and Critical COVID-19 Patients: A Retrospective, Single-Center Study. Front Med (Lausanne). 2021;8:585851. Published 2021 May 26. doi:10.3389/fmed.2021.585851

58. Berry DC, O'Byrne SM, Vreeland AC, Blaner WS, Noy N (August 2012). "Cross talk between signaling and vitamin A transport by the retinol-binding protein receptor STRA6". Molecular and Cellular Biology. 32 (15): 3164-75.

59. Dhokia, V., Macip, S. A master of all trades - linking retinoids to different signalling pathways through the multi-purpose receptor STRA6. Cell Death Discov. 7, 358 (2021). https://doi.org/10.1038/s41420-021-00754-z.

60. D.W. Clark. Aldehyde dehydrogenases in cancer stem cells: potential as therapeutic targets. Ann. Transl. Med., 4 (24) (2016), p. 518

61. Croker BA, Kiu H, Nicholson SE. SOCS regulation of the JAK/STAT signalling pathway. Semin Cell Dev Biol. 2008;19(4):414-422. doi:10.1016/j.semcdb.2008.07.010

62. Johnson HM, Lewin AS, Ahmed CM. SOCS, Intrinsic Virulence Factors, and Treatment of COVID-19. Front Immunol. 2020 Oct 23;11:582102. doi: 10.3389/fimmu.2020.582102. PMID: 33193390; PMCID: PMC7644869.

63. Hyperactivation of STAT3 occurs in COVID-19 infection and other viral infections. Matsuyama T, Kubli SP, Yoshinaga SK, Pfeffer K, Mak TW (2020). "An aberrant STAT pathway is central to COVID-19". Cell Death Differ. 27 (12): 3209-3225.

64. Barrett, Crisha et al. "Human Peripheral Blood Mononuclear Cells Express High Levels of the Vitamin a Transport Protein, Stimulated by Retinoic Acid 6 (P19-004-19)." Current Developments in Nutrition vol. 3,Suppl 1 nzz049.P19-004-19. 13 Jun. 2019, doi:10.1093/cdn/nzz049.P19-004-19

65. Chang F, Lee JT, Navolanic PM, Steelman LS, Shelton JG, Blalock WL, Franklin RA, McCubrey JA. Involvement of PI3K/Akt pathway in cell cycle progression, apoptosis, and neoplastic transformation: a target for cancer chemotherapy. Leukemia. 2003 Mar;17(3):590-603. doi: 10.1038/sj.leu.2402824. PMID: 12646949.

66. Sobierajska K, Fabczak H, Fabczak S. Kinazy receptorów sprzezonych z białkami G--ich regulacja i funkcja w komórce [Mechanisms of regulation and function of G-protein coupled receptor kinases]. Postepy Biochem. 2005;51(4):421-9. Polish. 
67. A. Yen, M.S. Roberson, S. Varvayanis, A.T. Lee. Retinoic acid induced mitogen-activated protein (MAP)/extracellular signal-regulated kinase (ERK) kinase-dependent MAP kinase activation needed to elicit HL-60 cell differentiation and growth arrest. Cancer Res., 58 (14) (1998), pp. 3163-3172

68. Z. Al Tanoury, A. Piskunov, C. Rochette-Egly. Vitamin a and retinoid signaling: genomic and nongenomic effects. J. Lipid Res., 54 (7) (2013), pp. 1761-177533. Jewell JL, Fu V, Hong AW, et al. GPCR signaling inhibits mTORC1 via PKA phosphorylation of Raptor. Elife. 2019;8:e43038. Published 2019 May 21.

69. Ferguson SS. Phosphorylation-independent attenuation of GPCR signalling. Trends Pharmacol Sci. 2007 Apr;28(4):173-9.

70. Aoto J, Nam Cl, Poon MM, Ting P, Chen L. Synaptic signaling by all-trans retinoic acid in homeostatic synaptic plasticity. Neuron. 2008 Oct 23;60(2):308-20.

71. Jewell JL, Fu V, Hong AW, Yu FX, Meng D, Melick CH, Wang H, Lam WM, Yuan HX, Taylor SS, Guan KL. GPCR signaling inhibits mTORC1 via PKA phosphorylation of Raptor. Elife. 2019 May 21;8:e43038.

72. Li JY, Liao CH, Wang Q, Tan YJ, Luo R, Qiu Y, Ge XY. The ORF6, ORF8 and nucleocapsid proteins of SARS-CoV-2 inhibit type I interferon signaling pathway. Virus Res. 2020 Sep;286:198074.

73. Matsuyama T, Kubli SP, Yoshinaga SK, Pfeffer K, Mak TW. An aberrant STAT pathway is central to COVID-19. Cell Death Differ. 2020 Dec;27(12):3209-3225.

74. Cabezas-Wallscheid N, Buettner F, Sommerkamp P, Klimmeck D, Ladel L, Thalheimer FB, PastorFlores D, Roma LP, Renders S, Zeisberger P, Przybylla A, Schönberger K, Scognamiglio R, Altamura S, Florian CM, Fawaz $M$, Vonficht $D$, Tesio $M$, Collier $P$, Pavlinic $D$, Geiger $H$, Schroeder T, Benes V, Dick TP, Rieger MA, Stegle O, Trumpp A. Vitamin A-Retinoic Acid Signaling Regulates Hematopoietic Stem Cell Dormancy. Cell. 2017 May 18;169(5):807-823.e19.

75. Anwar MM, Badawi AM, Eltablawy NA. Can the coronavirus infection penetrates the brain resulting in sudden anosmia followed by severe neurological disorders? eNeurologicalSci. 2020 Dec;21:100290.

76. Singh Y, Gupta G, Satija S, et al. COVID-19 transmission through host cell directed network of GPCR. Drug Development Research. 2020 Sep;81(6):647-649.

77. Abdel Hameid R, Cormet-Boyaka E, Kuebler WM, Uddin M, Berdiev BK. SARS-CoV-2 may hijack GPCR signaling pathways to dysregulate lung ion and fluid transport. Am J Physiol Lung Cell Mol Physiol. 2021 Mar 1;320(3):L430-L435.

78. Li Y, Wang W, Parker W, Clancy JP. Adenosine regulation of cystic fibrosis transmembrane conductance regulator through prostenoids in airway epithelia. Am J Respir Cell Mol Biol 34: 600-608, 2006.

79. Cahill TJ 3rd, Thomsen AR, Tarrasch JT, Plouffe B, Nguyen AH, Yang F, Huang LY, Kahsai AW, Bassoni DL, Gavino BJ, Lamerdin JE, Triest S, Shukla AK, Berger B, Little J 4th, Antar A, Blanc A, Qu CX, Chen X, Kawakami K, Inoue A, Aoki J, Steyaert J, Sun JP, Bouvier M, Skiniotis G, Lefkowitz RJ. Distinct conformations of GPCR- $\beta$-arrestin complexes mediate desensitization, signaling, and endocytosis. Proc Natl Acad Sci U S A. 2017 Mar 7;114(10):2562-2567. 
80. Baum A, García-Sastre A. Differential recognition of viral RNA by RIG-I. Virulence. 2011 MarApr;2(2):166-9.

81. Jockers R, Gbahou F, Tadagaki K, Kamal M. Oligomérisation des protéines humaines et virales à sept domaines transmembranaires - Nouvelle stratégie virale pour manipuler la cellule hôte [Oligomerization of human and viral 7TM proteins: a new viral strategy to manipulate host cells]. Med Sci (Paris). 2012 Oct;28(10):864-9. French.

82. Yamada T, Sato S, Sotoyama Y, Orba Y, Sawa H, Yamauchi H, Sasaki M, Takaoka A. RIG-I triggers a signaling-abortive anti-SARS-CoV-2 defense in human lung cells. Nat Immunol. 2021 Jul;22(7):820-828. doi: 10.1038/s41590-021-00942-0. Epub 2021 May 11. PMID: 33976430.

83. Zheng Y, Zhuang MW, Han L, Zhang J, Nan ML, Zhan P, Kang D, Liu X, Gao C, Wang PH. Severe acute respiratory syndrome coronavirus 2 (SARS-CoV-2) membrane (M) protein inhibits type I and III interferon production by targeting RIG-I/MDA-5 signaling. Signal Transduct Target Ther. 2020 Dec 28;5(1):299. doi: 10.1038/s41392-020-00438-7. PMID: 33372174; PMCID: PMC7768267.

84. N. Jamal, S. Whittier, R.C. Carter, P. Zachariah. Biphasic variation over time in presenting features of patients with COVID-19. Pediatrics, 146 (5) (2020)

84. Natalia Teruel, Matthew Crown, Matthew Bashton, Rafael Najmanovich. Computational analysis of the effect of SARS-CoV-2 variant Omicron Spike protein mutations on dynamics, ACE2 binding and propensity for immune escape. Natalia Teruel, Matthew Crown, Matthew Bashton, Rafael Najmanovich. bioRxiv 2021.12.14.472622; doi: https://doi.org/10.1101/2021.12.14.472622

85. Maren Schubert, Federico Bertoglio, Stephan Steinke, Philip Alexander Heine, Mario Alberto YngaDurand et.al. Human serum from SARS-CoV-2 vaccinated and COVID-19 patients shows reduced binding to the RBD of SARS-CoV-2 Omicron variant. medRxiv 2021.12.10.21267523; doi: https://doi.org/10.1101/2021.12.10.21267523

87. Callaway E. Heavily mutated Omicron variant puts scientists on alert. Nature. 2021 Dec;600(7887):21.

88. Meng B, Abdullahi A, Ferreira IATM, Goonawardane N, Saito A, Kimura I, Yamasoba D, Gerber PP, Fatihi S, Rathore S, Zepeda SK, Papa G, Kemp SA, Ikeda T, Toyoda M, Tan TS, Kuramochi J, Mitsunaga S, Ueno T, Shirakawa K, Takaori-Kondo A, Brevini T, Mallery DL, Charles OJ; CITIID-NIHR BioResource COVID- Collaboration; Genotype to Phenotype Japan (GP-Japan) Consortium members; EcuadorCOVID19 Consortium, Bowen JE, Joshi A, Walls AC, Jackson L, Martin D, Smith KGC, Bradley J, Briggs JAG, Choi J, Madissoon E, Meyer K, Mlcochova P, Ceron-Gutierrez L, Doffinger R, Teichmann SA, Fisher AJ, Pizzuto MS, de Marco A, Corti D, Hosmillo M, Lee JH, James LC, Thukral L, Veesler D, Sigal A, Sampaziotis F, Goodfellow IG, Matheson NJ, Sato K, Gupta RK. Altered TMPRSS2 usage by SARS-CoV-2 Omicron impacts tropism and fusogenicity. Nature. 2022 Feb 1. 